\title{
Relational event-time in quantum mechanics
}

\author{
Sebastian Fortin*, Olimpia Lombardi*, and Matías Pasqualini**1 \\ ${ }^{*}$ CONICET - Universidad de Buenos Aires \\ ** CONICET - Universidad Nacional de Rosario
}

\begin{abstract}
Some authors, inspired by the theoretical requirements for the formulation of a quantum theory of gravity, proposed a relational reconstruction of the quantum parametertime - the time of the unitary evolution, which would make quantum mechanics compatible with relativity. The aim of the present work is to follow the lead of those relational programs by proposing a relational reconstruction of the event-time-which orders the detection of the definite values of the system's observables. Such a reconstruction will be based on the modalHamiltonian interpretation of quantum mechanics, which provides a clear criterion to select which observables acquire a definite value and to specify in what situation they do so.
\end{abstract}

\section{Introduction}

Although the idea that time has a relational nature appeared already at the dawn of philosophy, it was with Leibniz that it acquired a more precise formulation that made it possible to discuss the ontology of time in the context of physical knowledge. Initially the discussion was overshadowed by the great success of Newtonian mechanics, with its assumption of absolute space and time; but later, various heirs of the Leibnizian perspective returned to defend the relational character of time. However, in general the debate between substantialism and relationalism remained confined to the realm of classical and relativistic mechanics, while the question about the nature of time was not a central topic in the highly developed field of the interpretation of quantum mechanics.

Nevertheless, some authors, inspired by the theoretical requirements for the formulation of a quantum theory of gravity, proposed a relational reconstruction of quantum time, which would make quantum mechanics compatible with general relativity (Page and Wootters 1983, Wootters 1984, Rovelli 1990, 1991, 2008). But there are two notions of time in quantum mechanics: a parameter-time, through which the system unitarily evolves, and an event-time,

\footnotetext{
${ }^{1}$ The three authors have contributed to the article to the same extent. The order of the names is alphabetical.
} 
which orders the detection of definite values of the system's observables. Those relational views point to a reconstruction of quantum mechanics that lacks the parameter-time as a fundamental item: the temporality of the evolution emerges as a relational result within the closed system itself, in particular, the universe as a whole.

The aim of the present work is to follow the lead of those relational programs by proposing a relational reconstruction also of the event-time. With this purpose, we will appeal to the modal-Hamiltonian interpretation (MHI) (Lombardi and Castagnino 2008, 2010, Castagnino and Lombardi 2008, Ardenghi, Castagnino, and Lombardi 2009), which provides a clear criterion to select which observables acquire a definite value and to specify in what situation they do so. In this way, the two notions of time in quantum mechanics emerge as a result of the internal relations defined in a closed quantum system.

In the light of this purpose, the paper is organized as follows. In Section 2 we will briefly review how the relational conception of time has been presented in both the philosophical and the physical fields. In Section 3 we will recall the peculiarities of time in quantum mechanics, stressing the relationship between time and energy and the two notions of time involved in the theory. Two relational programs about the parameter-time will be briefly and conceptually described in Section 4. Section 5 will be devoted to the presentation of the MHI, focusing on the relevant aspects for the discussion about the nature of the eventtime, in particular, the central role played by the Hamiltonian. In this interpretive context, in Section 6 we will show how the event-time can be constructed on the basis of the Hamiltonian structure itself, illustrating the proposal with the model of consecutive measurements. Finally, in Section 7 the proposal will be summarized, and the possible extrapolation of the event-time construction to the case of the Relational Quantum Mechanics will be considered.

\section{Relational time in philosophy and physics}

The traditional presentations of the relational views of space and time usually begin by reminding Gottfried Leibniz. However, the idea of a relational time goes back much further. Aristotle already conceived of time (although not space) in relational terms. For him, the natural world is the realm of change: everything that is natural is subject to change; and although change and time are intimately related, he explained time in terms of change and not vice versa. For him, "there is no time apart from change...." (Aristotle, Physics, Chapter 11) because "time is the measure of change" (Aristotle, Physics, Chapter 12) (see Barnes 1984). 
The next important step toward relational time is, certainly, Leibniz. In his well-known debate with Samuel Clarke -Isaac Newton's spokesman- in the 18th century (see Alexander 1956), Leibniz argued that space and time do not subsist independently of physical objects, but rather emerge as systems of relationships that take place between objects (relative distances in the case of space; relative durations in the case of time). One of Leibniz's arguments depends on the metaphysical principle of the identity of indiscernibles: given that a reference frame at rest and any other reference frame moving at constant speed (with respect to a supposed absolute space) generate equivalent descriptions, the notions of absolute space and time must be removed from the ontology since metaphysically superfluous.

The success of Newtonian theory, based on the assumption of an absolute space that made it possible to account for acceleration and rotation, left the Leibnizian vision in a lagging place in the context of physics. It was not until the end of the 19th century that Ernst Mach returned to Leibniz's relationalist ideas, in the search of a mechanical theory formulated only in terms of the relationships between bodies. According to Mach (1883), dynamic effects such as centrifugal forces on rotating objects are a consequence of the relative motion of objects with respect to the mass distribution of the universe and not with respect to absolute space. This view gave rise to what was later christened as "Mach's Principle" by Albert Einstein (for a detailed discussion of the several distinct ways of formulating the general idea of "Mach's Principle" that Einstein adopted at various times, see Hoefer 1995).

Although Mach focused his criticism on Newton's postulation of absolute space, he also wished to eliminate absolute time, so obtaining a completely relationalist version of classical mechanics. Such a version would be provided, many years later, by Julian Barbour and Bruno Bertotti, who faced the problem in a Lagrangian framework, invoking only relative magnitudes. After a first theory that was not perfectly equivalent to Newton's theory (Barbour and Bertotti 1977), the authors proposed a relational Lagrangian mechanics based on what they called "best matching," which can be viewed as a relational substitute for the principle of least action. By starting from two instantaneous relational configurations of the bodies in the universe, an "intrinsic difference" parameter is defined, which measures how different the two configurations are. The dynamically admissible trajectories are those that minimize that intrinsic difference (Barbour and Bertotti 1982; see also Barbour 1982). This reformulation predicts that the universe will have zero angular momentum and total energy that can be made zero, as is to be expected in a universe where space and time are completely relational (for an accurate analysis of this proposal, see Pooley and Brown 2002). 
When the strategy of best matching is transferred to relativity theories (see, e.g., Barbour 1999), however, the relationalist stance is not straightforward. Already in special relativity, the idea of instantaneous relational configurations of the entire universe stands in tension with relative simultaneity. In general relativity, in turn, best matching requires the foliability of space-time, and this is not a property of all possible models of Einstein field equations; moreover, if a space-time is foliable, in general there are many different possible foliations that define different instantaneous relational configurations of the entire universe (see Butterfield 2001). For these reasons, Barbour's relational views, when applied to general relativity, have been taken with some skepticism. For instance, it has been claimed that general relativity, if cast in its $3+1$, geometrodynamical form, can be considered a relational theory about time but not about space, and that this fact does not imply, nevertheless, that general relativity can also be viewed as a relational/Machian theory (Pooley 2001).

Whereas the debates about the relational nature of time in classical and relativistic mechanics have been many and varied, the question about the nature of time in quantum mechanics has not received the same attention, especially when compared to the large amount of literature on interpretive issues. Perhaps a contributing factor to this situation is the dual character of the notion of time in quantum mechanics.

\section{Time in quantum mechanics}

The symmetry group of quantum mechanics, the Galilean group, is the same as that of classical mechanics. Perhaps the invariance under the same symmetry group led many to think that the issue of the substantial or relational character of space and time in nonrelativistic quantum mechanics is automatically settled by the same discussion in prerelativistic classical mechanics. However, this view overlooks the peculiarities of the notion of time in quantum mechanics.

\subsection{Parameter-time and event-time}

In classical mechanics, the state of a system is defined in terms of the actual properties that the system acquires over time. In quantum mechanics, on the other hand, the state only provides probabilities on the possible values of the properties of the system, while such properties acquire current values only at some particular times (this view excludes the socalled "wavefunction realism", according to which the wavefunction is a physical field on a 
high-dimensional space; see, e.g., Albert 2013). Therefore, at least two different notions of time need to be distinguished in the quantum realm (see Rovelli 1991, Busch 2008):

- The parameter-time (also called 'dynamical' or 'intrinsic' time by Busch 2008) is the time along which the system unitarily evolves. It is represented by the variable $t$ as it appears in the Schrödinger equation.

- The event-time (also called 'observable' time by Busch 2008) is the time at which individual events occur. Those events are measurement results or, more generally, any acquisition of a definite value by a certain observable.

The parameter-time is the notion of time involved in the characterization of the Galilean group; in quantum mechanics it is supposed to be homogeneous and isotropic as in the classical case. The Schrödinger equation rules how the probabilities on the possible values of all the observables of the system change along the parameter-time. The event-time, on the contrary, has no formal representation in quantum mechanics. Nevertheless, it is essential in order to endow the theory with physical meaning: testing the theory is only possible by means of the registration of specific events, such as the hit of an electron on a screen or the absorption of a photon by an atom, occurring at particular times.

An additional feature of standard quantum mechanics is that the formalism of the theory does not establish any link between the parameter-time and the event-time. The quantum state only assigns a probability to each possible event and the Schrödinger equation gives the evolution of those probabilities along the parameter-time. However, there is no theoretical specification about when an actual event occurs. These facts are related to the obstacles to define an observable time, as will be considered in the following subsection.

\subsection{Time and energy}

On the basis of the Fourier analysis of wave packets, "uncertainty" relations between position and wave number (spatial frequency), and between time and (time) frequency, can be formulated: $\Delta x \Delta k \geq 2 \pi, \Delta t \Delta \omega \geq 2 \pi$. By appealing to the de Broglie relations, $p=\hbar k$ and $E=\hbar \omega$, Niels Bohr (1928) proposed uncertainty relations for position-momentum and timeenergy with the same status:

$$
\Delta x \Delta p \geq h \quad \Delta t \Delta E \geq h .
$$


Since then, the debates about the meaning of the time-energy uncertainty relation became ubiquitous in the quantum foundations literature, with no general agreement (for a thorough discussion, see Busch 1990a, 1990b).

The main obstacle to conceive the uncertainty relation for position-momentum and the uncertainty relation for time-energy on an equal footing is that, whereas position, momentum, and energy are represented by operators, time is represented by a scalar; in other words, time is not an observable of the quantum system. The natural strategy to overcome this difficulty would be to define a time observable $T$ that would allow obtaining the uncertainty relation for time-energy as a particular case of the general relation between any two observables $A$ and $B$ :

$$
\Delta_{\rho} A \cdot \Delta_{\rho} B \geq \frac{1}{2}\left|\langle[A, B]\rangle_{\rho}\right|=\frac{1}{2}\left|\langle A B-B A\rangle_{\rho}\right|,
$$

where $\rho$ is the system's state $\left(\rho=|\psi\rangle\langle\psi|\right.$ when the state is pure), and $\Delta_{\rho} O^{2}=\left\langle O^{2}\right\rangle_{\rho}-\langle O\rangle_{\rho}^{2}$. In fact, this was the idea of Werner Heisenberg (1927), who, in his famous paper on uncertainty relations, defined a time operator $T$, conjugate to the Hamiltonian $H$, and formulated the corresponding uncertainty relation. However, some years later, Wolfgang Pauli (1933) proved a theorem according to which the fact that any Hamiltonian is bounded from below precludes the existence of a self-adjoint operator $T$ acting as a generator of a unitary group representation of translations in the energy spectrum.

Given the limitation imposed by Pauli's theorem, the time-energy uncertainty relation was formulated by appealing to a generic observable $R$, incompatible with energy, which acts as an observable correlate of time (Mandelstam and Tamm 1945; for simple presentations, see Messiah 1961, Ballentine 1989). The characteristic time $\tau_{R}$ is defined as

$$
\tau_{R}=\frac{\Delta_{\rho} R}{\left|\frac{d}{d t}\langle R\rangle_{\rho}\right|} .
$$

By combining the Heisenberg equation $i \hbar d R / d t=[A, H]$ with the general uncertainty relation of eq. (2), the definition of $\tau_{R}$ leads to

$$
\tau_{R} \Delta_{\rho} H \geq \hbar / 2 .
$$

However, the characteristic time $\tau_{R}$ cannot be considered a perfect substitute for time $t$, since it depends on the particular observable $R$. 
Beyond the large amount of work on this topic, what is important here is how the uncertainty relation for time-energy relates to the two notions of time. When conceived in Bohr's terms, or even in terms of a characteristic time, the uncertainty relation involves the parameter-time, that is, the time on which the wavefunction (the quantum state) evolves (or the observables evolve in the Heisenberg picture). On the other hand, the impossibility of defining a self-adjoint operator $T$ representing a time observable is the manifestation of the absence of the theoretical characterization of the event-time in quantum mechanics. This difference between the two notions of time will be relevant for the proposal of a relational reconstruction of quantum time.

\section{Relational parameter-time in quantum mechanics}

Although comparatively much less discussed than in classical and relativistic mechanics, the question about the nature of time in quantum mechanics has also been considered. Some authors focused on the characterization of a relational quantum time in order to pave the way for quantum gravity. In fact, as many specialists stress, one of the main obstacles to the formulation of a quantum theory of gravity is the difference between the notions of time in quantum theory and in general relativity, a theory invariant under general coordinate transformations. In Chris Isham's words:

A key ingredient in all these questions [the questions involved in the so-called 'the problem of time in quantum gravity'] is the realisation that the notion of time used in conventional quantum theory is grounded firmly in Newtonian physics. Newtonian time is a fixed structure, external to the system: a concept that is manifestly incompatible with diffeomorphism-invariance and also with the idea of constructing a quantum theory of a truly closed system (such as the universe itself). (Isham 1993: 6; see also Kuchař 1991).

In the face of this situation, the idea of reducing quantum time through a relational construction appears as a promising solution.

An interesting proposal in this direction is that put forward by Don Page and William Wootters with their "evolution without evolution" (1983; see also Wootters 1984). Their central idea is that the universe is a stationary scenario, and that time and evolution emerge in a subsystem of the universe, which is entangled with another subsystem that fulfills the conditions to be considered a clock. More precisely (for a simple presentation, see Marletto and Vedral 2017), the universe is assumed to be in a stationary state such that $H|\psi\rangle=0$ 
(which is in agreement with the Wheeler-De Witt equation for a closed universe, see De Witt 1967). It is also assumed that there is a system $S_{C}$ that can play the role of a clock: it has a large set of distinguishable states $|t\rangle$ and it ideally does not interact with the rest of the universe $S_{R}$ :

$$
H=H_{C} \otimes I_{R}+I_{C} \otimes H_{R},
$$

where $H_{C}$ and $H_{R}$ are the Hamiltonians and $I_{C}$ and $I_{R}$ are the identities of $S_{C}$ and $S_{R}$ respectively. Moreover, the conjugate observable $T_{C}$ to the clock Hamiltonian, $\left[H_{C}, T_{C}\right]=i$, with $T_{C}|t\rangle=t|t\rangle$, is defined. Despite not interacting with each other, the clock $S_{C}$ and the rest of the universe $S_{R}$ are supposed to be entangled in the sense that

$$
|\psi\rangle=\sum_{t} \alpha_{t}|t\rangle \otimes\left|\phi_{t}\right\rangle
$$

where the $\left|\phi_{t}\right\rangle$ correspond to $S_{R}$ and $\alpha_{t} \neq 0$ for some $t$ (ideally, for a very large number of $t$ ). If the evolution of the clock is given by $|t\rangle=\exp \left(-i H_{C} t\right)|0\rangle$, where $|0\rangle$ is an eigenstate of $T_{C}$ taken as an initial time, then the history of the rest of the universe $S_{R}$ is given by the sequence of the correlated $\left|\phi_{t}\right\rangle$. In turn, when the value of the observable $T_{C}$ of the clock $S_{C}$ is $t$, the relative state, in the Everett (1957) sense, of $S_{R}$ is $\rho_{t}=\left|\phi_{t}\right\rangle\left\langle\phi_{t}\right|$. And given that $S_{C}$ and $S_{R}$ do not interact (see eq. (5)), the evolution of the rest of the universe with respect to the parameter $t$ takes the Schrödinger form:

$$
\frac{\partial \rho_{t}}{\partial t}=i\left[\rho_{t}, H_{R}\right]
$$

On this basis, Wootters concludes that

it is not necessary to include 'time' as an a priori element of physics. Clock time, which makes sense even within a stationary state, is the only kind of time that can be observed. Furthermore, even the existence of clock time is only a contingent property of the world [...]. Only certain states of the universe admit such a universal time. (Wootters 1984: 710).

A different but equally interesting approach to relational time in quantum mechanics is that advanced by Carlo Rovelli $(1990,1991,2008)$. The first step of this proposal is to let aside the Schrödinger picture of quantum mechanics, in which states evolve and observables are time-independent, and to embrace the Heisenberg picture, according to which what evolves in time are the observables $A$ and not the state $|\psi\rangle$ :

$$
i \hbar \frac{d A(t)}{d t}=[A(t), H]
$$


In this picture, the state $|\psi\rangle$ does not change, it is time-independent (which is in agreement with the Wheeler-De Witt equation, $H|\psi\rangle=0$ ). From this perspective, observables at different times are, strictly speaking, different observables. On this basis, Rovelli generalizes the Heisenberg picture by distinguishing between partial observables and complete observables (Rovelli 2002). A partial observable is a quantity to which a measuring procedure leading to a number can be associated; it is represented by such a procedure. A complete observable is a quantity whose probability distribution can be predicted by the theory; it is represented by an operator. In the generic case, a complete observable is composed of several partial observables. For example, the determination of the value of the electric field at a given space-time point, $E(\boldsymbol{x}, t)$, requires five measuring instruments: one electric field detector that measures $E$, three distance measuring devices giving the three components of $\boldsymbol{x}$, and a clock measuring $t$. Therefore, $E(\boldsymbol{x}, t)$ is a complete observable composed of five partial observables: $E$, the three components of $\boldsymbol{x}$, and $t$.

From Rovelli's perspective, the different partial observables should be conceived on the same footing: time is a partial observable with no privilege over the others. This means that dynamics expresses the relation between partial observables, as in the case of general relativity. If the unitary evolution of the partial observables with respect to a given specific partial observable is to be recovered, this last one has to satisfy certain requirements that make it to be called 'clock'. But clocks may not exist for a particular system, or may exist only in particular circumstances. This idea can be expressed in mathematical terms. The set of the complete observables of a system has a structure. In certain cases, this structure can be foliated in such a way that the resulting leaves are one-dimensional sets containing all the "Heisenberg observables" $A\left(t_{1}\right), A\left(t_{2}\right), A\left(t_{3}\right), \ldots$ corresponding to the same "Schrödinger observable" $A$. Or, in Rovelli's language, if the set can be foliated into one-parameter families that are given by the same partial observables with respect to another particular partial observable, this last one can be conceived as a clock that measures the time evolution of the partial observables of the system. In this case, it is said that the set of complete observables has a time structure. However, it may happen that, although dynamics always expresses the relation between partial observables, there is no such a time structure. In this sense, Rovelli agrees with Page and Wootters's conclusion: the existence of time is only a contingent property of the universe.

Both the programs of Page and Wootters and of Rovelli intend to show how and under which circumstances the time of the quantum unitary evolution (in the Schrödinger picture in 
the first case and in the Heisenberg picture in the second case) can be reconstructed from correlations. In other words, the authors focus on the emergence of the parameter-time, but say nothing about the event-time. As Rovelli explicitly acknowledges:

There are two independent notions of time in ordinary quantum mechanics: the time in which the system evolves, and the "time" that orders the measurements of the observer. These two are not related and may be non-coincident. I am talking here about the time in which the system evolves, not about the time of the observer. (Rovelli 1991: 130).

This program would be completed if a relational reconstruction of the quantum event-time could be formulated. But, as explained above, the event-time has no formal representation in quantum mechanics. When and how observables acquire definite values is a question that depends on how the theory is interpreted. For this reason, we will appeal to the modalHamiltonian interpretation to reconstruct the event-time, since this interpretation provides a clear criterion to select the observables that acquire definite values and to specify in what situation they do so.

\section{The modal-Hamiltonian interpretation}

The roots of the modal interpretations can be found in the works of Bas van Fraassen (1972, 1973, 1974), who distinguished between which physical properties the system may possess and what actually is the case. On the basis of this original idea, in the 1980s several authors presented realist "modal" interpretations (for an overview and references, see Dieks and Vermaas 1998) which, despite differences among them, agree on the following points (see Lombardi and Dieks 2021):

- The interpretation is based on the standard formalism of quantum mechanics without the projection postulate.

- The interpretation is realist in the sense that (i) it aims at describing how reality would be if quantum mechanics were true, and (ii) it assumes that quantum systems always possess a number of definite properties.

- Quantum mechanics is a fundamental theory, which must describe not only elementary particles but also macroscopic objects.

- Quantum mechanics describes single systems: the quantum state refers to a single system, not to an ensemble of systems. 
- The quantum state of the system (pure or mixed) describes its possible properties and not its actual properties. The relationship between the quantum state and the actual properties is probabilistic.

- A quantum measurement is an ordinary physical interaction. There is no collapse of the quantum state, which always evolves unitarily according to the Schrödinger equation.

As it is well-known, the Kochen-Specker theorem (Kochen and Specker 1967) proves the contextuality of quantum mechanics, that is, the impossibility of ascribing precise values to all the observables of a quantum system simultaneously, while preserving the functional relations between observables. Therefore, realist no-collapse interpretations are committed to selecting a privileged set of definite-valued observables out of all observables. Each modal interpretation thus supplies an "actualization rule" that picks out, from the set of all the observables of a quantum system, the subset of definite-valued properties that constitutes the preferred context.

\subsection{The postulates of the interpretation}

The modal-Hamiltonian interpretation of quantum mechanics (MHI) (Lombardi and Castagnino 2008, Castagnino and Lombardi 2008, Lombardi 2018), which belongs to the modal family, endows the Hamiltonian of the quantum system with a determining role, both in the definition of systems and subsystems and in the selection of the preferred context.

The MHI is based on the following postulates:

Systems postulate (SP): A quantum system $S$ is represented by a pair $(\mathcal{O}, H)$ such that (i) $\mathcal{O}$ is a space of self-adjoint operators on a Hilbert space $\mathcal{H}$, representing the observables of the system, (ii) $H \in \mathcal{O}$ is the time-independent Hamiltonian of the system $S$, and (iii) if $\rho_{0} \in \mathcal{O}^{\prime}$ (where $\mathcal{O}^{\prime}$ is the dual space of $\mathcal{O}$ ) is the initial state of $S$, it evolves according to the Schrödinger equation in its von Neumann version.

Although any quantum system can be decomposed in parts in many ways, according to the MHI a decomposition leads to parts which are also quantum systems only when the components' behaviors are dynamically independent of each other, that is, when there is no interaction among the subsystems:

Composite systems postulate (CSP): A quantum system represented by $S:(\mathcal{O}, H)$ is composite when it can be partitioned into two quantum systems 
represented by $S^{1}:\left(\mathcal{O}^{1}, H^{1}\right)$ and $S^{2}:\left(\mathcal{O}^{2}, H^{2}\right)$ such that (i) $\mathcal{O}=\mathcal{O}^{1} \otimes \mathcal{O}^{2}$, and (ii) $H=H^{1} \otimes I^{2}+I^{1} \otimes H^{2}$, (where $I^{1}$ and $I^{2}$ are the identity operators in the corresponding tensor product spaces). In this case, we say that $S^{1}$ and $S^{2}$ are subsystems of the composite system, $S=S^{1} \cup S^{2}$. If the system is not composite, it is elemental.

Regarding the preferred context, the central idea of the MHI is that the Hamiltonian of the system defines actualization. Any observable that does not commute with and/or does not have the symmetries of the Hamiltonian cannot acquire a definite actual value, since this actualization would break the symmetry of the system in an arbitrary way:

Actualization rule (AR): Given an elemental quantum system represented by $S:(\mathcal{O}, H)$ the actual-valued observables of $S$ are $H$ and all the observables commuting with $H$ and having, at least, the same symmetries as $H$.

This actualization rule has been applied to many well-known physical situations, such as the free particle with spin, the harmonic oscillator, the free hydrogen atom, the Zeeman effect, the fine structure, and the Born-Oppenheimer approximation, leading to results consistent with experimental evidence (see Lombardi and Castagnino 2008, Section 5). The MHI has extended its applications to further situations, such as the no-collapse account of consecutive measurements in physics (Ardenghi, Lombardi, and Narvaja 2013) and the problem of optical isomerism in chemistry (Fortin, Lombardi, and Martínez González 2018). The interpretation has been presented under a Galilean-invariant form, in terms of the Casimir operators of the Galilean group (Ardenghi, Castagnino, and Lombardi 2009, Lombardi, Castagnino, and Ardenghi 2010). It has also been argued that the MHI actualization rule can be transferred to the relativistic domain by changing the symmetry group accordingly: the definite-valued observables of a system would be those represented by the Casimir operators of the Poincare group, which are the mass operator and the squared spin operator (Ardenghi, Castagnino, and Lombardi 2011, Lombardi and Fortin 2015). This conclusion is in agreement with a usual assumption in quantum field theory: elemental particles always have definite values of mass and spin, and those values are precisely what define the different kinds of elemental particles of the theory. From an ontological viewpoint, the MHI proposes an ontology of properties, lacking the ontological category of individual (da Costa, Lombardi, and Lastiri 2013, da Costa and Lombardi 2014), which supplies an adequate answer to the problem of entanglement of indistinguishable systems (Fortin and Lombardi 2021), but does not prevent the emergence of particles under particular circumstances (Lombardi and Dieks 
2016). In this paper we will not discuss these aspects of the MHI, but will only focus on the answer to the measurement problem that it supplies.

\subsection{Quantum measurement}

A quantum single measurement (the difference between single measurement, frequency measurement, and state measurement is explained in Lombardi and Castagnino 2008, Section 6) is a three-stage process:

- First stage $(I)$ : The system $S:\left(\mathcal{O}^{S}, H^{S}\right)$ to be measured and the measuring apparatus $M:\left(\mathcal{O}^{M}, H^{M}\right)$ do not interact. Therefore, they are elemental subsystems of the composite system $U^{(I)}:\left(\mathcal{O}^{(I)}, H^{(I)}\right)$, where $\mathcal{O}^{(I)}=\mathcal{O}^{S} \otimes \mathcal{O}^{M}$ and $H^{(I)}=H^{S} \otimes I^{M}+I^{S} \otimes H^{M}$. The system $S$ is in a state $\sum_{i=1}^{n} c_{i}\left|a_{i}\right\rangle$, where the $\left|a_{i}\right\rangle$ are the eigenstates of an observable $A$ of $S$, and the apparatus $M$ is in a ready-to-measure state $\left|p_{0}\right\rangle$, eigenstate of the pointer $P$ of $M$. Thus, the state of $U^{(I)}$ in this stage is

$$
\left|\Psi^{(I)}\right\rangle=\left(\sum_{i=1}^{n} c_{i}\left|a_{i}\right\rangle\right) \otimes\left|p_{0}\right\rangle .
$$

- Second stage (II): $S$ and $M$ interact through an interaction Hamiltonian $H^{\text {int }}$ that introduces a correlation between the eigenstates $\left|a_{i}\right\rangle$ of $A$ and the eigenstates $\left|p_{i}\right\rangle$ of $P$. Therefore, the whole system becomes the elemental system $U^{(I I)}:\left(\mathcal{O}^{(I I)}, H^{(I I)}\right)$, where $\mathcal{O}^{(I I)}=\mathcal{O}^{(I)}$ and $H^{(I I)}=H^{S} \otimes I^{M}+I^{S} \otimes H^{M}+H^{\text {int }}$, and whose state is

$$
\left|\Psi^{(I I)}\right\rangle=\sum_{i=1}^{n} c_{i}\left|a_{i}\right\rangle \otimes\left|p_{i}\right\rangle
$$

- Third stage (III): The interaction ends and the whole system is again composite, $U^{(I I I)}=U^{(I)}: S$ and $M$ become elemental systems as in the first stage. The state in this stage is $\left|\Psi^{(I I I)}\right\rangle=\left|\Psi^{(I I)}\right\rangle$.

The measurement problem consists in explaining why the pointer $P$ of the apparatus $M$ acquires a definite value in stage III. When applied to $M$, the MHI actualization rule states that the definite-valued observables are the Hamiltonian $H^{M}$ and all the observables commuting with $H^{M}$ and having, at least, its same symmetries -degeneracies. According to the MHI, $P$ is a definite-valued observable because $P$ commutes with $H^{M}$ and does not break its symmetries. These features of $P$ are not required to make the MHI work, but are supported by plausible physical reasons. In fact, on the one hand, for the reading of the pointer to be possible, the eigenvectors $\left|p_{i}\right\rangle$ of $P$ have to be stationary, that is, $\left[P, H^{M}\right]=0$. On the other hand, in practice the apparatus is a macroscopic system, whose Hamiltonian is 
the result of the interaction among a huge number of degrees of freedom. Since, in general, symmetries are broken by interactions, the symmetry of the Hamiltonian decreases with the complexity of the system. Then, a macroscopic system having a Hamiltonian with symmetries is a highly exceptional situation: in the generic case, energy is the only constant of motion of the macroscopic system. As a consequence, in practice $H^{M}$ is non-degenerate, and the set of its eigenstates is a basis of the Hilbert space $\mathcal{H}^{M}$ of the apparatus. But the pointer $P$ must not have a so huge number of eigenvalues as $H^{M}$, because the experimental physicists have to be able to discriminate among them. This means that, in general, $P$ is a "collective" observable (see Omnés 1994, 1999), that is, a highly degenerate observable whose eigenprojectors introduce a sort of "coarse-graining" in $\mathcal{H}^{M}$. In summary, given that $\left[P, H^{M}\right]=0$, and since $P$ has more degeneracies than $H^{M}$ (which is non-degenerate), $P$ will be a definite-valued observable.

This MHI answer to the measurement problem has proved to be able to supply an account of the problem both in its ideal and its non-ideal versions, overcoming some wellknown criticisms to the original modal interpretations (see Albert and Loewer 1990, 1993). In particular, it was applied to the Stern-Gerlach experiment taking into account the possibility of infinite "tails" (Elby 1993). Moreover, in the non-ideal case it gives a criterion to distinguish between reliable and non-reliable measurements (Lombardi and Castagnino 2008: Section 6), a criterion that can be generalized when expressed in informational terms (Lombardi, Fortin, and López 2015).

According to the collapse interpretation, in each particular detection, the pure state $\left|\Psi^{(I I)}\right\rangle$ of eq. (10) indeterministically "collapses" to a component of the superposition, say, $\left|a_{k}\right\rangle \otimes\left|p_{k}\right\rangle$, which is interpreted as that the system $S$ is in the state $\left|a_{k}\right\rangle$ and the apparatus $M$ is in the state $\left|p_{k}\right\rangle$ with certainty; in other words, as that the observable $A$ of $S$ and the pointer $P$ of $M$ acquire the definite values $a_{k}$ and $p_{k}$, respectively. The collapse hypothesis gives a straightforward account of the agreement between the outcomes of consecutive measurements of the same observable. In fact, a second measurement of the observable $A$ on the system $S$ will maintain the system in the same state $\left|a_{k}\right\rangle$ resulting from the collapse due to the first measurement. This means that the conditional probability of obtaining the value $p_{k}^{(2)}$ of the pointer $P^{(2)}$ of the apparatus $M^{(2)}$ in the second measurement, given that the value $p_{k}^{(1)}$ of $P^{(1)}$ of $M^{(1)}$ was obtained in the first measurement, is $\operatorname{pr}\left(p_{k}^{(2)} / p_{k}^{(1)}\right)=1$. The collapse hypothesis also supplies a simple explanation to the non-trivial correlations between the outcomes of consecutive measurements of different observables (see, e.g., Cohen- 
Tannoudji et al. 1977). As a consequence, any no-collapse interpretation is committed to explain the correlations in consecutive measurements in an empirically adequate way.

In the case of the MHI, it can be shown that the correlations between the outcomes of consecutive measurements can be accounted for without collapse (see Ardenghi, Lombardi, and Narvaja 2013). Now measurement is a five-stage process:

- First stage $(I)$ : The system $S:\left(\mathcal{O}^{S}, H^{S}\right)$ to be measured and the measuring apparatuses $M^{(1)}:\left(\mathcal{O}^{M^{(1)}}, H^{M^{(1)}}\right)$ and $M^{(2)}:\left(\mathcal{O}^{M^{(2)}}, H^{M^{(2)}}\right)$ do not interact. Therefore, they are elemental subsystems of the composite system $U^{(I)}:\left(\mathcal{O}^{(I)}, H^{(I)}\right)$, where $\mathcal{O}^{(I)}=\mathcal{O}^{S} \otimes \mathcal{O}^{M^{(1)}} \otimes \mathcal{O}^{M^{(2)}}$ and $H^{(I)}=H^{S} \otimes I^{M^{(1)}} \otimes I^{M^{(2)}}+I^{S} \otimes H^{M^{(1)}} \otimes I^{M^{(2)}}+I^{S} \otimes I^{M^{(1)}} \otimes H^{M^{(2)}}$. In this case, the apparatuses $M^{(1)}$ and $M^{(2)}$ are in the ready-to-measure states $\left|p_{0}^{(1)}\right\rangle$ and $\left|p_{0}^{(2)}\right\rangle$, eigenstates of the pointers $P^{(1)}$ of $M^{(1)}$ and $P^{(2)}$ of $M^{(2)}$, respectively. Thus, the state of $U^{(I)}$ in this stage is

$$
\left|\Psi^{(I)}\right\rangle=\left(\sum_{i=1}^{n} c_{i}\left|a_{i}\right\rangle\right) \otimes\left|p_{0}^{(1)}\right\rangle \otimes\left|p_{0}^{(2)}\right\rangle .
$$

- Second stage (II): $S$ and $M^{(1)}$ interact through an interaction Hamiltonian $H^{\mathrm{int} S M^{(1)}}$ that introduces a correlation between the eigenstates $\left|a_{i}\right\rangle$ of $A$ and the eigenstates $\left|p_{i}^{(1)}\right\rangle$ of $P^{(1)}$. Therefore, now there are two elemental systems: $S M^{(1)}=\left(\mathcal{O}^{S M^{(1)}}, H^{S M^{(1)}}\right)$, where $\mathcal{O}^{S M^{(1)}}=\mathcal{O}^{S} \otimes \mathcal{O}^{M^{(1)}} \quad$ and $\quad H^{S M^{(1)}}=H^{S} \otimes I^{M^{(1)}}+I^{S} \otimes H^{M^{(1)}}+H^{\mathrm{int} S M^{(1)}}, \quad$ and $M^{(2)}:\left(\mathcal{O}^{M^{(2)}}, H^{M^{(2)}}\right)$. So, the whole system becomes $U^{(I I)}:\left(\mathcal{O}^{(I I)}, H^{(I I)}\right)$, where $\mathcal{O}^{(I I)}=\mathcal{O}^{(I)}=\mathcal{O}^{S M^{(1)}} \otimes \mathcal{O}^{M^{(2)}}$ and $H^{(I I)}=H^{S M^{(1)}} \otimes I^{M^{(2)}}+I^{S M^{(1)}} \otimes H^{M^{(2)}}$, and whose state is

$$
\left|\Psi^{(I I)}\right\rangle=\sum_{i=1}^{n} c_{i}\left|a_{i}\right\rangle \otimes\left|p_{i}^{(1)}\right\rangle \otimes\left|p_{0}^{(2)}\right\rangle .
$$

- Third stage (III): The first interaction ends and the whole system is again composite, $U^{(I I I)}=U^{(I)}$, in a state $\left|\Psi^{(I I I)}\right\rangle=\left|\Psi^{(I I)}\right\rangle$.

- Fourth stage (IV): $S$ and $M^{(2)}$ interact through an interaction Hamiltonian $H^{\text {int } S M^{(2)}}$ that introduces a correlation between the eigenstates $\left|a_{i}\right\rangle$ of $A$ and the eigenstates $\left|p_{i}^{(2)}\right\rangle$ of $P^{(2)}$. Therefore, now there are two elemental systems: $M^{(1)}:\left(\mathcal{O}^{M^{(1)}}, H^{M^{(1)}}\right)$ and $S M^{(2)}=\left(\mathcal{O}^{S M^{(2)}}, H^{S M^{(2)}}\right), \quad$ where $\quad \mathcal{O}^{S M^{(2)}}=\mathcal{O}^{S} \otimes \mathcal{O}^{M^{(2)}} \quad$ and $H^{S M^{(2)}}=H^{S} \otimes I^{M^{(2)}}+I^{S} \otimes H^{M^{(2)}}+H^{\text {int } S M^{(2)}}$. So, the whole system becomes $U^{(I V)}:\left(\mathcal{O}^{(I V)}, H^{(I V)}\right), \quad \quad$ where $\quad \mathcal{O}^{(I V)}=\mathcal{O}^{(I)}=\mathcal{O}^{S M^{(2)}} \otimes \mathcal{O}^{M^{(1)}} \quad$ and $H^{(I V)}=H^{S M^{(2)}} \otimes I^{M^{(1)}}+I^{S M^{(2)}} \otimes H^{M^{(1)}}$, and whose state is

$$
\left|\Psi^{(I V)}\right\rangle=\sum_{i=1}^{n} c_{i}\left|a_{i}\right\rangle \otimes\left|p_{i}^{(1)}\right\rangle \otimes\left|p_{i}^{(2)}\right\rangle \text {. }
$$


- Fifth stage $(V)$ : The second interaction ends and the whole system is again composite, $U^{(V)}=U^{(I)}: S, M^{(1)}$, and $M^{(2)}$ become elemental systems as in the first stage. The state in this stage is $\left|\Psi^{(V)}\right\rangle=\left|\Psi^{(I V)}\right\rangle$.

In stages III and $\mathrm{V}, P^{(1)}$ and $P^{(2)}$ acquire definite values, respectively, because they commute respectively with $H^{M^{(1)}}$ and $H^{M^{(2)}}$ and do not break their symmetries, as explained in the case of single measurement. But in this case, the correlations in the state of the whole system at the final stage explain the consistency between the measurements of the apparatuses $M^{(1)}$ and $M^{(2)}$. In fact, on the basis of state $\left|\Psi^{(V)}\right\rangle=\left|\Psi^{(I V)}\right\rangle$, the probability that $M^{(2)}$ measures $p_{k}^{(2)}$ given that $M^{(1)}$ has measured $p_{k}^{(1)}$ can be computed as

$$
\operatorname{pr}\left(p_{k}^{(2)} \mid p_{k}^{(1)}\right)=\frac{\operatorname{pr}\left(p_{k}^{(2)} \wedge p_{k}^{(1)}\right)}{\operatorname{pr}\left(p_{k}^{(1)}\right)}=\frac{\left|c_{k}\right|^{2}}{\left|c_{k}\right|^{2}}=1,
$$

yielding the expected correlation. It is worth stressing that $\operatorname{pr}\left(p_{k}^{(2)} \wedge p_{k}^{(1)}\right)$ is a legitimate probability since the pointers $P^{(1)}$ and $P^{(2)}$ are commuting observables because, in general, they belong to different measuring apparatuses which, as a consequence, are represented by different Hilbert spaces (see Laura and Vanni 2008). This result can be generalized for consecutive measurements of different observables and for any number of measurements.

In summary, consecutive measurements can be adequately explained without collapse if the perspective of the whole system is taken seriously: reduced states are not used as representing the component systems, but the state of the complete system is always retained in such a way that it codifies all possible correlations. In the next section we will see how the event-time arises as the consecuence of the interaction relations internal to the whole system (typically, the universe as a whole).

\section{A relational event-time for quantum mechanics}

\subsection{When observables acquire definite values}

Any interpretation that postulates the actualization of certain facts (the acquisition of definite values by observables) as a not merely epistemic but objective phenomenon is committed to specifying when actualization occurs. In the traditional versions of the Copenhagen interpretation, the collapse of the wavefunction is conceived as a sort of actualization linked to the act of measurement: collapse happens when the quantum system interacts with a macroscopic device or when a conscious being becomes aware of the result of the measurement (see Faye 2019). In the GRW version of quantum mechanics (Ghirardi, Rimini, 
and Weber 1986), collapse is a physical indeterministic phenomenon that repeatedly and spontaneously occurs with a probability $1 / \tau$ per second, where $\tau$ is a new constant of nature. According to some versions of the many-worlds interpretation, every measurement or, in general, every interaction splits the universe into myriad of copies of itself (see, e.g., DeWitt 1970). In the MHI interpretation, according to which actualization is an objective physical fact, the problem of deciding when such a fact occurs also cries for an answer. As explained below, a simple solution can be given when the definition of the preferred context is taken into account.

It is interesting to compare the MHI interpretation with other modal interpretations with respect to this point. For instance, in the biorthogonal-decomposition and the spectraldecomposition interpretations (see Lombardi and Dieks 2021), the preferred context depends on the instantaneous state of the system, which continuously changes over time. This means that actualization is a phenomenon that repeatedly occurs at each instant. This interpretational position leads to the need to account for the dynamics of actual properties (see Vermaas 1996). In the MHI interpretation, on the contrary, this step is unnecessary since the dynamics of actual properties is trivial. In fact, the preferred context where actualization occurs is not a function of time, since it depends on the Hamiltonian: the definite-valued observables always commute with the Hamiltonian and, therefore, they are constants of motion of the system. In other words, the observables that receive definite values are the same during all the "life" of the quantum system as such - precisely, as a closed system-: they do not change from the time of the constitution of the system -due to the interaction between other systems or as the result of the cessation of an interaction- up to the time when the system disappears -by interacting with another system or by decomposing into different subsystems when an interaction ends.

The idea that certain properties of a physical system, such as its energy, change only upon interaction is very natural in the classical realm. Nevertheless, quantum mechanics introduces peculiarities that need to be taken into account: due contextuality, not all possible properties can have definite actual values simultaneously. In the face of this essential quantum feature, different interpretations adopt diverse positions about actualization and, as commented above, according to some of them certain properties of a quantum system acquire definite actual values independently of interactions. By contrast, the MHI agrees with the natural intuition that interactions, when begin and end, lead to changes (this claim will be accurately illustrated in the model of the next subsection). Summing up, actualization occurs only once in a quantum system, at the time of its constitution as a closed system, and from 
that time there is no change in the domain of actuality until the system disappears: the definite-valued observables with their corresponding definite actual values remain unmodified during the system's entire life, delimited by interaction events.

The MHI gives then a precise definition of which observables acquire definite values by means of its actualization rule- and of when these precise events occur -when the system is constituted as a closed system. This view can be naturally related to the difference between parameter-time and event-time:

- The parameter-time is the evolution time of the state according the Schrödinger equation. Since the quantum state encodes probabilities and not actuality, the parameter-time is the time along which the propensities (in Popper's terminology) or potentialities (in Heisenberg's terminology) to actualization of all the observables of the system evolve. To the extent that there is no collapse, these potentialities keep changing unitarily throughout the parameter-time with no discontinuities.

- The event-time does not correspond to the change of the quantum state, but is the time defined by the actualization of the system's properties belonging to the preferred context. In other words, since events are actual and not merely possible facts, the event-time belongs to the realm of actuality and results from the relations between events. Moreover, according to this view:

- Since the event-time is constituted by the relations between events, there is no event-time between events.

- Since events are given by the interaction relations between systems, the event-time is a discrete time that emerges from those interaction relations.

\subsection{Event-time from interactions}

The interactions between systems are usually conceived as ordered in time. For instance, in the previous section consecutive measurements were described in this way, that is, as different stages that are consecutive regarding parameter-time. However, if the event-time is to be relationally constructed, that strategy is not legitimate. But this is not an obstacle, because the interactions involved in consecutive measurements can be described in a temporally neutral way, in terms of the corresponding events, that is, of the acquisition of definite values by the observables of the preferred context.

Let us represent the event that the observable $X$ acquires a definite value $x$ with " $(X: x)$ ". From an atemporal perspective, then, there are only events. If for simplicity only 
the events related to the Hamiltonian are considered (the events referred to the rest of the observables of the preferred context are implicit), the events involved in the consecutive measurements described in the previous section are:

- Event $\left(H^{S M^{(1)}}: \omega_{k}^{S M^{(1)}}\right)$ : The elemental system $S M^{(1)}$ is constituted by the interaction between the system $S$ and the apparatus $M^{(1)}$, so its Hamiltonian $H^{S M^{(1)}}$ acquires a definite value, say, $\omega_{k}^{S M^{(1)}}$.

- Events $\left(H^{S}: \omega_{k}^{S}\right)$ and $\left(H^{M^{(1)}}: \omega_{k}^{M^{(1)}}\right)$ : The elemental systems $S$ and $M^{(1)}$ are constituted by the end of the interaction between them, so their Hamiltonians $H^{S}$ and $H^{M^{(1)}}$ acquire definite value, say, $\omega_{k}^{S}$ and $\omega_{k}^{M^{(1)}}$, respectively.

- Event $\left(H^{S M^{(2)}}: \omega_{k}^{S M^{(2)}}\right)$ : The elemental system $S M^{(2)}$ is constituted by the interaction between the system $S$ and the apparatus $M^{(2)}$, so its Hamiltonian $H^{S M^{(2)}}$ acquires a definite value, say, $\omega_{k}^{S M^{(2)}}$.

- Events $\left(H^{S}: \omega_{k}^{S}\right)$ and $\left(H^{M^{(2)}}: \omega_{k}^{M^{(2)}}\right)$ : The elemental systems $S$ and $M^{(2)}$ are constituted by the end of the interaction between them, so their Hamiltonians $H^{S}$ and $H^{M^{(2)}}$ acquire definite value, say, $\omega_{k}^{S}$ and $\omega_{k}^{M^{(1)}}$, respectively.

If the instants of the event-time are given by the events in themselves, four instants can be identified, which can be conventionally called:

- $\tau_{1}$ : the event-instant associated with event $\left(H^{S M^{(1)}}: \omega_{k}^{S M^{(1)}}\right)$.

$-\tau_{2}$ : the event-instant associated with events $\left(H^{S}: \omega_{k}^{S}\right)$ and $\left(H^{M^{(1)}}: \omega_{k}^{M^{(1)}}\right)$.

$-\tau_{3}:$ the event-instant associated with event $\left(H^{S M^{(2)}}: \omega_{k}^{S M^{(2)}}\right)$.

$-\tau_{4}$ : the event-instant associated with events $\left(H^{S}: \omega_{k}^{S}\right)$ and $\left(H^{M^{(2)}}: \omega_{k}^{M^{(2)}}\right)$.

As it is now quite clear, the relations between those event-instants are the result of the web of interaction relations between the involved systems: interactions (let us call them "Int") and ends of interactions (let us call them “ $\neg$ Int") give rise to a net of events from which the event-time emerges. In the case of the above example of consecutive measurements, such a net can be described as in Figure 1.

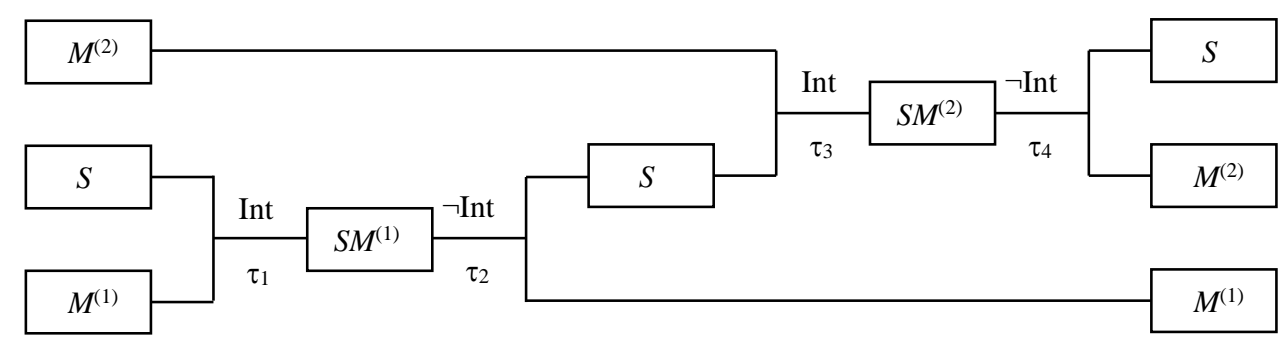


Figure 1: Net of interaction relations, events and event-time instants.

At this point it could be asked about what "imprints" directionality to the so constituted event-time. The natural answer is that the interaction relations Int and $\neg$ Int are essentially directional phenomena that give rise to a directional network, which endows the emerging event-time with directionality. Although natural, this answer presupposes the existence of a privileged direction of time embodied in those interaction relations. But such a presupposition is far from trivial: the problem of the arrow of time is one of the traditional issues in the foundations of physics. The traditional view, which defines the future as the direction of time in which the entropy of isolated systems increases, does not seem to be appropriate in this case: here interactions are primitive and entropic considerations play no role. Nevertheless, a non-entropic approach can be adopted: according to John Earman's "time direction heresy" (Earman 1974), the arrow of time is an intrinsic property of time (or of space-time), which does not need to be reduced to non-temporal features. Following Earman's program, the arrow can be defined exclusively in terms of the structure of time (or of space-time) itself (although from the perspective of general relativity, this is the line followed in Castagnino, Lombardi, and Lara 2003, Castagnino, Lara, and Lombardi 2003, Castagnino and Lombardi 2004, 2009). In this case, the difference between the two directions of time would be given by the asymmetry of the structure of the network of interaction events from which the event-time arises. Of course, the issue of the directionality of the event-time is not settled by these brief comments, but requires a discussion that goes beyond the limits of the present paper. Here the point to stress is that time directionality does not require entropic considerations, but can emerge from the properties of the very structure of events, as does the event-time itself.

\subsection{Event-time in a closed universe}

As explained, the MHI actualization rule applies to closed systems, and the preferred context is defined by the Hamiltonian of the whole system. In turn, actualization occurs when the system is constituted as such, and since then, to the extent that it remains closed, the eventtime does not "pass". But, what about the universe as a whole?

The above description might lead to suggest that in the universe, the closed system par excellence, there is no event-time. However, this is not the case. Let us recall that the MHI actualization rule applies to elemental closed systems, that is, non-interacting systems that 
cannot be further analyzed into subsystems. Therefore, nothing prevents elemental systems from existing as subsystems of a closed system like the universe, and whose interaction relations establish the network from which the event-time emerges. For instance, let us consider the above example of consecutive measurements as a closed toy-model of the universe (see Figure 1): the internal structure of the interaction relations between the subsystems of the whole universe is sufficient to generate the event-time.

However, somebody might reply that, whereas the Hamiltonian of the universe is unique and time-independent -as in the Wheeler-De Witt equation-, the total Hamiltonian of the consecutive-measurement toy-model changes from stage to stage (see the description of the five stages in Subsection 5.2). The usual interpretation is that, in such a model, the Hamiltonian is a function of the continuous parameter-time: first, say from $t_{0}$ to $t_{1}$, there are no interactions; at $t_{1}$ and until $t_{2}, S$ and $M^{(1)}$ interact through the interaction Hamiltonian $H^{\text {int } S M^{(1)}}$; from $t_{2}$ until $t_{3}$, that interaction ceases; at $t_{3}$ and until $t_{4}, S$ and $M^{(2)}$ interact through $H^{\mathrm{int} S M^{(2)}}$; and, finally, from $t_{4}$, the interaction ceases again. However, from a coherent relational view about time, the parameter-time must be also relational and, as a consequence, this orthodox account is not legitimate.

One strategy, perhaps the theoretically most elegant one, would be to reconstruct the parameter-time from the approaches of Page and Wootters or of Rovelli (recall Section 4). In this case, a particular system -in Page and Wootters's proposal- or a particular partial observable -in Rovelli's proposal- that can play the role of a "clock" should be found, in terms of which the unitary evolution of the system could be recovered and the different stages of our toy-model could be defined. But since in the context of our problem -the relational reconstruction of the event-time- the purpose is not to recover that unitary evolution but only to describe the changes of the Hamiltonian in atemporal terms, the strategy can be much easier. In fact, the total Hamiltonian can retain its time-independence if its "changes" are expressed in function of another, non-temporal variable.

In the case of our consecutive-measurement toy-model, the selection of the nontemporal variable and the construction of the time-independent total Hamiltonian seem natural. In order to perform the consecutive measurements, the measuring apparatuses have to be arranged in very precise spatial dispositions on a table and under very specific conditions. Let us call this system 'measuring arrangement' $M^{T}$, whose parts are the different measuring apparatuses $M^{(1)}$ and $M^{(2)}$. Therefore, the system $S$ only interacts with $M^{T}$, composing a single toy-universe $U=S M^{T}$, whose Hamiltonian $H^{U}$ includes the self-Hamiltonians of the 
subsystems and all the interaction Hamiltonians, now expressed in terms of the spatial disposition of the subsystems. In the case of our toy-model,

$$
\begin{aligned}
H^{U}= & H^{S} \otimes I^{M^{(1)}} \otimes I^{M^{(2)}}+I^{S} \otimes H^{M^{(1)}} \otimes I^{M^{(2)}}+I^{S} \otimes I^{M^{(1)}} \otimes H^{M^{(2)}}+ \\
& H^{\mathrm{int} S M^{(1)}} \otimes I^{M^{(2)}}+H^{\mathrm{int} S M^{(2)}} \otimes I^{M^{(1)}}
\end{aligned}
$$

The interaction Hamiltonians have the following form:

$$
H^{\mathrm{int} S M^{(1)}}=\Theta(q) H^{S M^{(1)}} \quad H^{\mathrm{int} S M^{(2)}}=\Omega(q) H^{S M^{(2)}},
$$

where $\Theta(q)$ and $\Omega(q)$ are double-step functions,

$$
\Theta(q)=\left\{\begin{array}{l}
0 \text { if } q<q_{1} \\
1 \text { if } q_{1} \leq q \leq q_{2} \\
0 \text { if } q>q_{2}
\end{array} \quad \Omega(q)=\left\{\begin{array}{l}
0 \text { if } q<q_{3} \\
1 \text { if } q_{3} \leq q \leq q_{4}, \\
0 \text { if } q>q_{4}
\end{array}\right.\right.
$$

with $q_{2}<q_{3}$. In this way, the space variable $q$, which is linked to the disposition of the elements of the measuring arrangement, replaces the parameter-time $t$ in the ordering of the interaction relations in the very inside of the toy-universe.

Of course, this consecutive-measurement toy-universe is an extremely simplified model. Nevertheless, it contains all the conceptual elements necessary to show how the event-time arises from the network of interaction relations between the systems that compose the whole closed universe, and how the structure of that event-time is embodied in the internal structure of the Hamiltonian of that universe.

\subsection{Time and order}

As explained in the previous subsections, the event-time emerges from the network of interaction relations between the subsystems of the universe, and its directionality results from the directionality of those relations: if systems $S_{A}$ and $S_{B}$ interact yielding $S_{C}$, the relation does not hold in reverse direction; if inside the system $S_{D}$ an interaction ends leading to systems $S_{E}$ and $S_{F}$, the relation neither holds in reverse direction. Therefore, the start and the end of interactions establish a strict order relation in the set of events, that is, a relation that is irreflexive, asymmetric, and transitive. And given that the instants of the event-time are defined by the events themselves, this strict order relation is translated to the set of the instants of the event-time. For example, in the toy-model of the consecutive measurements described above, the strict order is $\tau_{1}<\tau_{2}<\tau_{3}<\tau_{4}$, where "<" represents the relation "being 
earlier than" (see Figure 1): the event-time with its order structure arises from the relations between events.

Nevertheless, the above toy-model is a simple case, in which all the events and eventinstants are arranged in a total order, that is, an order relation on a set that links any two members of the set. But other cases are possible; for example, it may be the case that two systems never interact again after a first interaction. In other words, in the generic case, events and event-instants are not ordered as in a chain. The instants of the event-time (and the corresponding events) are related by a partial order, since it is possible that, given two instants, neither of them is prior to the other, so that the relation "being earlier than" does not hold for them. For instance, the event-time might bifurcate into two different lines when two systems stop interacting and never interact again, generating event evolutions completely disconnected (see Figure 2a); or two lines of the event-time, coming from disconnected event evolutions, might converge when two systems interact and yield a single evolution (see Figure 2b).

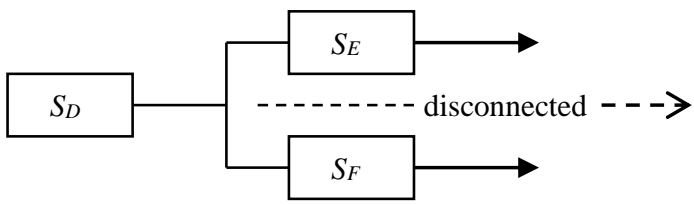

(a)

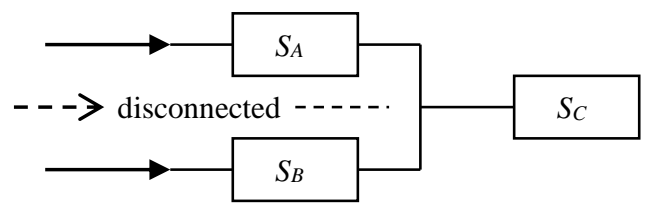

(b)

Figure 2: Examples of event-time generating a partial order.

The possibility of an event-time with a partial-order structure is at odds with the traditional notion of time of classical physics, that is, a "unilinear" time, "the same" for all the events of the universe. But, in principle, nothing prevents the event-time of quantum mechanics from being completely different from the classical time. The debate about how time order should be conceived has already arisen in the context of the construction of a relational time in other fields of physics.

In a relatively recent article about the problem of time in quantum gravity, Sean Gryb and Karim Thébault (2016) demur against the approaches based on a radical relational construction of time. In particular, they criticize what they call "the correlation, or partial observables, view of time" (2016: 665), closely associated with Rovelli's works. The authors describe such a relational position as claiming that "all that it is for a physical degree of freedom to change is for it to vary with regard to a second physical degree of freedom." 
(2016: 684); and they add that, according to the relational view, "[w]e are free to choose any degree of freedom as an internal clock, and such an arbitrarily chosen clock may, for some finite interval, give us a useful means of marking both the duration between, and order of, events as defined by correlations between the other degrees of freedom." (2016: 684). A point to note in this regard is that this presentation of Rovelli's proposal is not entirely fair, since the author is very clear in that not just any degree of freedom can be used as a clock: a partial observable must satisfy certain requirements to play the role of a clock, and it may be the case that those requirements are not fulfilled for a given system. The same remark holds for Page and Wootters's proposal, in which the existence of time is also a contingent fact.

Anyway, this is not the central point here. What is relevant in the present context is the criticism that Gryb and Thébault direct against Rovelli's partial-observables perspective, but which could also be applied to Page and Wootters's "evolution-without-evolution" view. According to the authors, the radical relational position about time "generally will not give us an ordering of events in 'time' that is globally defined in terms of a linear sequence" (Gryb and Thébault 2016: 684; italics added). This is a problem in the light of their own conception of what the very concept of time means: "Although we can recover a weak sense of change as relative variation, there is no scope for the basic one-dimensional ordering structure that, in our view, is constitutive of time." (2016: 666; italics added). Therefore, they propose a different relational approach, according to them Machian in nature, which leads to an absolute temporal-ordering structure: "for a notion of time to be relational in the Machian sense, it is not enough to be merely relational; it must also be unique and equitable" (2016: 686); "equitable in that it can be derived uniquely from the motions of the entire system taken together" (2016: 685).

In the quantum context, the target of Gryb and Thébault's criticism is the relational construction of the parameter-time. However, since the criticism is based on the fact that a time "globally defined in terms of a linear sequence" cannot be obtained, it can also be applied to the relational construction of the event-time as that proposed here. In fact, the so resulting event-time introduces a partial order between events and not a "one-dimensional ordering structure" as that required by the authors. The question is: Why should the relational time of quantum mechanics be linear and total-ordering? According to the authors' view, those features are constitutive of the very concept of time. However, such a view could be seen as a metaphysical prejudice in favor of a pre-relativistic concept of time. 
As it is well-known, in general relativity the concept of time appears under two different faces. On the one hand, the "coordinate time" is a coordinate on the space-time manifold and, therefore, it is expected to play no fundamental role in a theory that is invariant under general coordinate transformations (Isham 1993: 12). On the other hand, the "proper time" is the time measured along a certain world-line: it is linear and totally orders the events of such a worldline, but cannot temporally coordinate all the events of the universe. Only if the space-time admits a cosmic time, can the proper times corresponding to the world-lines of all the bodies of the universe be coordinated by a single time (see Castagnino, Lombardi, and Lara 2003), that is, by a time that is "unique and equitable" as required by Gryb and Thébault. But the existence of a cosmic time -and even the weaker condition of the existence of a global time (see Hawking and Ellis 1973), equivalent to the foliability of the space-time required by the authors- imposes a relevant constraint to the set of space-time models. Such a constraint, although reasonable for studying certain aspects of the observable universe, seems inadmissible in a discussion about the very foundations of time: concepts of time that "refer to the description of special solutions of the Einstein field equations only [...] are irrelevant in a discussion of the ontology of time, because a different ontology for different solutions of the same theory is certainly unsatisfactory" (Rovelli 2006: 34).

In summary, the discussion about the order structure of relational time in quantum mechanics should take into account the general motivation of the relational project. Precisely, in view of the peculiar features of general relativity, the fact that the resulting event-time does not have the structure of classical time should be considered as an advantage for the construction of a truly fundamental quantum gravity. In other words, if one of the goals of a relational reconstruction of time in quantum mechanics is to face the so-called, in Isham words, "problem of time in quantum gravity", then the possibility of a non unilinear eventtime, far from being an obstacle, should be welcome.

\section{Final remarks}

The aim of the present work has been to extend the program of a relational time for quantum mechanics, which Page and Wootters and Rovelli developed for the parameter-time, to the case of the event-time. With this purpose, we have appealed to the modal-Hamiltonian interpretation, because it provides a precise definition of the quantum events and of the situations in which they occur. We have thus shown how the event-time can be constructed on 
the basis of the structure of the Hamiltonian of the complete closed system, and we have illustrated the proposal with a universe toy-model based on consecutive measurements.

A final remark is in order. Rovelli's proposal of a relational time for quantum mechanics is in clear resonance with his Relational Quantum Mechanics (RQM) (Rovelli 1996; see also Laudisa and Rovelli 2021), according to which the properties of a system acquire definite values objectively but only relative to another system. The MHI, by contrast, endows a closed system with certain definite-valued non-relational properties. In other words, whereas in the RQM the events involving a system are always relative to another system, in the MHI an event is the acquisition of a definite value by an observable of a closed system, independently of any other system. This difference might lead one to think that these two views cannot harmonize with each other at all. However, this may not be the case. Despite that difference, the two interpretations agree in the following relevant points: (i) events are objective; (ii) events occur only in interactions and, therefore, they are discrete; (iii) measurements are ordinary physical interactions; (iv) the quantum state does not describe a physical field but supplies a codification of probabilities. On this basis, perhaps the two interpretive stances can be made compatible if applied at different levels: the MHI gives the perspective of the entire closed system, strictly speaking, the universe as a whole, whereas the RQM describes the objective and relative values acquired by the properties of the subsystems of the universe in their incessant interactions. Therefore, the construction of the event-time as proposed in the present paper might be adapted to the perspective of the RQM; but this will be the subject of a future work.

\section{Acknowledgements}

This work was supported by grant ID-61785 of the John Templeton Foundation and by grant PICT-04519 of the Agencia Nacional de Promoción Científica y Tecnológica of Argentina.

\section{References}

Albert, D. Z. (2013)."Wave function realism.” Pp. 1-51 in 58-75 in A. Ney and D. Z. Albert (eds.), The Wave Function: Essays on the Metaphysics of Quantum Mechanics. Oxford: Oxford University Press.

Albert, D. and Loewer, B. (1990). "Wanted dead or alive: Two attempts to solve Schrödinger's paradox." Pp. 277-285 in Proceedings of the 1990 Biennial Meeting of the Philosophy of Science Association, Vol. 1. East Lansing: Philosophy of Science Association. 
Albert, D. and Loewer, B. (1993). "Non-ideal measurements." Foundations of Physics Letters, 6: 297 305.

Alexander, H. G. (ed.) (1956). The Leibniz-Clarke Correspondence. Manchester, UK: Manchester University Press.

Ardenghi, J. S., Castagnino, M., and Lombardi, O. (2009). "Quantum mechanics: modal interpretation and Galilean transformations." Foundations of Physics, 39: 1023-1045.

Ardenghi, J. S., Castagnino, M., and Lombardi, O. (2009). "Modal-Hamiltonian interpretation of quantum mechanics and Casimir operators: the road to quantum field theory." International Journal of Theoretical Physics, 50: 774-791.

Ardenghi, J. S., Lombardi, O., and Narvaja, M. (2013). "Modal interpretations and consecutive measurements." Pp. 207-217 in V. Karakostas and D. Dieks (eds.), EPSA 2011: Perspectives and Foundational Problems in Philosophy of Science. Berlin: Springer.

Ballentine, L. (1989). Quantum Mechanics. A Modern Development. Singapore: World Scientific.

Barbour, J. (1982). "Relational concepts of space and time." The British Journal for the Philosophy of Science, 33: 251-274.

Barbour, J. B. (1999). The End of Time: The Next Revolution in Our Understanding of the Universe. London: Weidenfeld \& Nicholson.

Barbour, J. and Bertotti, B. (1977). "Gravity and inertia in a Machian framework." Nuovo Cimento, 38B: 1-27.

Barbour, J. and Bertotti, B. (1982). "Mach's Principle and the structure of dynamical theories." Proceedings of the Royal Society (London), 382: 295-306.

Barnes, J. (ed.) (1984). The Complete Works of Aristotle, Volumes I and II, Princeton: Princeton University Press.

Bohr, N. (1928). "Das Quantenpostulat und die neuere Entwicklung der Atomistik." Naturwissenschaften, 16: 245-257.

Butterfield, J. (2001). "The end of time?" British Journal for the Philosophy of Science, 53: 289-330.

Busch P. (1990a). "On the energy-time uncertainty relation. Part I: Dynamical time and time indeterminacy." Foundations of Physics, 20: 1-32.

Busch P. (1990b). "On the energy-time uncertainty relation. Part II: Pragmatic time versus energy Indeterminacy." Foundations of Physics, 20: 33-43.

Busch P. (2008). "The time-energy uncertainty relation." Pp. 73-105 in J. Muga, R. S. Mayato, and I. Egusquiza (eds.). Time in Quantum Mechanics. Lecture Notes in Physics, vol 734. Berlin-Heidelberg: Springer.

Castagnino, M., Lara, L., and Lombardi, O. (2003). "The cosmological origin of timeasymmetry." Classical and Quantum Gravity, 20: 369-391.

Castagnino, M. and Lombardi, O. (2004). "The generic nature of the global and non-entropic arrow of time and the double role of the energy-momentum tensor." Journal of Physics A (Mathematical and General), 37: 4445-4463. 
Castagnino, M. and Lombardi, O. (2008). "The role of the Hamiltonian in the interpretation of quantum mechanics.” Journal of Physics. Conferences Series, 28: \#012014.

Castagnino, M. and Lombardi, O. (2009). "The global non-entropic arrow of time: from global geometrical asymmetry to local energy flow." Synthese, 169: 1-25.

Castagnino, M., Lombardi, O., and Lara, L. (2003). "The global arrow of time as a geometrical property of the universe." Foundations of Physics, 33: 877-912.

Da Costa, N. and Lombardi, O. (2014). "Quantum mechanics: ontology without individuals." Foundations of Physics, 44: 1246-1257.

De Witt, B. (1970). “Quantum mechanics and reality.” Physics Today, 23: 30-35.

Cohen-Tannoudji, C., Diu, B., and Lalöe, F. (1977). Quantum Mechanics. New York: John Wiley \& Sons.

Da Costa, N., Lombardi, O., and Lastiri, M. (2013). "A modal ontology of properties for quantum mechanics". Synthese, 190: 3671-3693.

DeWitt, B. S. (1967). “Quantum theory of gravity.” Physical Review D, 160: 1113-1148.

Dieks, D. and Vermaas, P. E. (1998). The Modal Interpretation of Quantum Mechanics. Dordrecht: Kluwer Academis Publishers.

Earman, J. (1974). "An attempt to add a little direction to «the problem of the direction of time»." Philosophy of Science, 41: 15-47.

Elby, A. (1993). "Why 'modal' interpretations don't solve the measurement problem." Foundations of Physics Letters, 6: 5-19.

Everett, H. (1957). "Relative state formulation of quantum mechanics." Reviews of Modern Physics, 29: 454-462.

Faye, J. (2019). "Copenhagen interpretation of quantum mechanics.” In E. N. Zalta (ed.), Stanford Encyclopedia of Philosophy (Winter 2019 Edition). Stanford: Stanford University. https://plato.stanford.edu/entries/qm-copenhagen/

Fortin, S. and Lombardi, O. (2021). "Entanglement and indistinguishability in a quantum ontology of properties." Forthcoming in Studies in History and Philosophy of Science.

Fortin, S., Lombardi, O., and Martínez González, J. C. (2018). “A new application of the modal-Hamiltonian interpretation of quantum mechanics: the problem of optical isomerism." Studies in History and Philosophy of Modern Physics, 62: 123-135.

Ghirardi, GC., Rimini, A., and Weber, T. (1986). "Unified dynamics for microscopic and macroscopic systems", Physical Review D, 34: 470-491.

Gryb, S. and Thébault, K. (2016). “Time remains." The British Journal for the Philosophy of Science, 67: 663-705.

Heisenberg, W. (1927). "Über den anschaulichen Inhalt der quantentheoretischen Kinematik und Mechanik." Zeitschrift für Physik, 43: 172-198.

Hawking, S. and Ellis, G. (1973). The Large Scale Structure of Space-Time. Cambridge: Cambridge University Press.

Hoefer, C. (1995). “Einstein's formulations of Mach's Principle.” Pp. 67-87 in J. Barbour and H. Pfister (eds.), Mach's Principle. From Newton's Bucket to Quantum Gravity. Boston: Birkhäuser. 
Isham, C. J. (1993) "Canonical quantum gravity and the problem of time." Pp 157-287 in L. A. Ibort and M. A. Rodríguez (eds.), Integrable Systems, Quantum Groups, and Quantum Field Theories, NATO ASI Series (Series C: Mathematical and Physical Sciences), vol 409. Dordrecht: Springer (page number from arxiv.org/abs/grqc/9210011).

Kuchař, K. (1991). "The problem of time in canonical quantization." Pp. 141-171 in A. Ashtekar and J. Stachel (eds.), Conceptual Problems of Quantum Gravity. Boston: Birkhäuser.

Laudisa, F. and Rovelli, C. (2021). "Relational quantum mechanics." In E. N. Zalta (ed.), Stanford Encyclopedia of Philosophy (Spring 2021 Edition). Stanford: Stanford University. https://plato.stanford.edu/entries/qm-relational/.

Laura, R. and Vanni, L. (2008). "Conditional probabilities and collapse in quantum measurements." International Journal of Theoretical Physics, 47: 2382-2392.

Lombardi, O. (2018). "The Modal-Hamiltonian Interpretation: measurement, invariance and ontology." Pp. 32-50 in O. Lombardi, S. Fortin, C. López, and F. Holik (eds.), Quantum Worlds. Perspectives on the Ontology of Quantum Mechanics. Cambridge: Cambridge University Press.

Lombardi, O. and Castagnino, M. (2008). "A modal-Hamiltonian interpretation of quantum mechanics." Studies in History and Philosophy of Modern Physics, 39: 380-443.

Lombardi, O., Castagnino, M., and Ardenghi, J. S. (2010). "The modal-Hamiltonian interpretation and the Galilean covariance of quantum mechanics." Studies in History and Philosophy of Modern Physics, 41: 93-103.

Lombardi. O. and Dieks, D. (2016). "Particles in a quantum ontology of properties". Pp. 123143 in T. Bigaj and C. Wüthrich (eds.), Metaphysics in Contemporary Physics. Leiden: Brill-Rodopi.

Lombardi, O. and Dieks, D. (2021). "Modal interpretations of quantum mechanics." In E. N. Zalta (ed.), Stanford Encyclopedia of Philosophy (Winter 2021 Edition). Stanford: Stanford University. https://plato.stanford.edu/entries/qm-modal/.

Lombardi, O. and Fortin, S. (2015). "The role of symmetry in the interpretation of quantum mechanics." Electronic Journal of Theoretical Physics, 12: 255-272.

Lombardi, O., Fortin, S., and López, C. (2015). "Measurement, interpretation and information." Entropy, 17: 7310-7330.

Mach, E. (1883) [1960]. The Science of Mechanics: A critical and Historical Account of its Development, trans. by T. J. McCormack. La Salle: Open Court.

Mandelstam, L. and Tamm, I. (1945). "The uncertainty relation between energy and time in non-relativistic quantum mechanics.” Journal of Physics (USSR), 9: 249-254.

Marletto, C. and Vedral, V. (2017). "Evolution without evolution and without ambiguities." Physical Review D, 95: 043510.

Messiah, A. (1961). Quantum Mechanics, Vol. 1. Amsterdam: North-Holland.

Omnés, R. (1994). The Interpretation of Quantum Mechanics. Princeton: Princeton University Press.

Omnés, R. (1999). Understanding Quantum Mechanics. Princeton: Princeton University Press. 
Page, D. and Wootters, W. (1983). "Evolution without evolution.” Physical Review D, 27: 2885-2892.

Pauli (1933). "Die allgemeinen Prinzipien der Wellenmechanik." Pp. 83-272 in H. Geiger and K. Scheel (eds.), Handbuch der Physik, 2nd ed., Vol 24. Berlin: Springer-Verlag.

Pooley, O. (2001). "Relationalism rehabilitated? II: Relativity." PhilSci Archive, ID: 221. http://philsci-archive.pitt.edu/221/.

Pooley, O. and Brown, H. (2002). "Relationalism rehabilitated? I: Classical mechanics." The British Journal for the Philosophy of Science, 53: 183-204.

Rovelli, C. (1990). "Quantum mechanics without time: A model." Physical Review D, 42: 2638-2646.

Rovelli, C. (1991). "Is there incompatibility between the ways time is treated in general relativity and in standard quantum mechanics?" Pp. 126-136 in A. Ashtekar and J. Stachel (eds.), Conceptual Problems of Quantum Gravity. New York: Birkhauser.

Rovelli, C. (1996). "Relational quantum mechanics." International Journal of Theoretical Physics, 35: 1637-1678.

Rovelli, C. (2002). “Partial observables.” Physical Review D, 65: \#124013.

Rovelli, C. (2006). "The disappearance of space and time.” Pp. 25-35 in D. Dieks (ed.), The Ontology of Spacetime, Amsterdam: Elsevier.

Rovelli, C. (2008). "Forget time." Essay written for the FQXi contest on the Nature of Time.

Van Fraassen, B. C. (1972). “A formal approach to the philosophy of science.” Pp. 303-366 in R. Colodny (ed.), Paradigms and Paradoxes: The Philosophical Challenge of the Quantum Domain. Pittsburgh: University of Pittsburgh Press.

Van Fraassen, B. C. (1973). "Semantic analysis of quantum logic." Pp. 80-113 in C. A. Hooker (ed.), Contemporary Research in the Foundations and Philosophy of Quantum Theory. Dordrecht: Reidel.

Van Fraassen, B. C. (1974). “The Einstein-Podolsky-Rosen paradox.” Synthese, 29: 291-309.

Vermaas, P. E. (1996). "Unique transition probabilities in the modal interpretation.\# Studies in History and Philosophy of Modern Physics, 27: 133-159.

Wootters, W. (1984). "“Time" replaced by quantum correlations.” International Journal of Theoretical Physics, 23: 701-711. 